\title{
ONTOLOGY TRANSLATION: THE SEMIOTIC ENGINEER- ING OF CONTENT MANAGEMENT SYSTEMS
}

\author{
ALEJANDRO VILLAMARIN M.
}

The present paper proposes the application of Semiotic Engineering theory to Content Management Systems (CMS) focusing on the analysis of how the use of different ontologies can affect the user's efficiency when performing tasks in a CMS. The analysis is performed using the theoretical semiotic model Web-Semiotic Interface Design Evaluation (W-SIDE model).

The Ontology Translation concept is proposed as a practical enhancement for the W-SIDE model. The enhanced model is validated by using a proof of concept based on test cases of a fictitious scenario. The validation process is carried out modifying an open source CMS. The testing of the modified CMS if performed by a volunteer tester that fits both the Ontology Translation concept and the fictitious scenario. Finally, the results of the experiment are critically evaluated establishing limitations, conclusions, and recommendations for future studies. 


\section{INTRODUCTION}

It is argued by Villamarin (2015) that content management systems must be seen not just as commercial products than can be traded. They should be considered as complex interactive communication tools. According to Armour (2000), software is a medium to store knowledge and its value is not in the code itself, but what it does. At the same time, De Souza et al. (2001) points out that despite all the theoretical frameworks that have been used to analyse the human factor dimension in artefacts, simple questions still remain without a satisfactory answer. For instance, how can a theoretical framework support the design of user interface languages?

In this context, the present research applies semiotic theory to the content management interface of a CMS. This is in order to answer the following question: How can a CMS be guaranteed to be "easy to use" if rather than being an ad-hoc tool it is a general purpose tool?

\section{PRELIMINARIES}

\subsection{SEMIOTIC ENGINEERING - THE DIGITAL COMMUNICATION PROCESS}

De Souza (1993) argues that computational systems have two intrinsic communicative roles: they can send and receive messages at the immediate interface level, but at the same time they can be seen as messages sent from a user interface designer to users using the computational medium. Therefore, it is argued that systems can be considered as metacommunication artefacts. Semiotic Engineering constitutes a theoretical background that helps to study the Human-Computer Interaction where systems are considered metacommunication artefacts.

The concept of semiotic engineering is used by Valtolina et al. (2012) to define a digital communication process model, which is composed of the following elements:

- Two communicants: an end-user (11) and an interactive system designer (12). 12 is not presented during the process of interaction between 11 and the software system involved in the communication process.

- A software system, the decoding process performed by users is carried out through interaction with the software system developed by 12 (the designer).

- A message $\alpha$, which is exchanged by means of the computer hardware. Consequently, it is virtual, transient, dynamic, and multi-modal. The persistence of the message is: (a) perpetual for its internal form and (b) transient for its external form (i.e. its materialisation exists as long as a software program interprets and materialises it).

\subsection{W-SIDE FRAMEWORK}

The Web-Semitic Interface Design Evaluation Framework (W-SIDE Framework) is proposed by Speroni (2006) as a set of conceptual utilities for analysing the semantics of web interface elements. Its main purpose is the evaluation of the correspondence between the knowledge presupposed by web interfaces and the one owned by critical users. It is argued by Speroni (2006) that the role of interface designers is to understand what the website should talk about and how the user (based on their experience) can interact with it. The core of the W-SIDE Framework is the W-SIDE Model. This model is composed of seven concepts: 
I. Web Semiotic Unit - It is a sign (group of strongly interrelated signs) used to compose a meaningful and functional message to the user.

II. Conceptual Semantics - It links the semiotic unit to pre-existing knowledge of the user regarding the topic the website talks about, i.e. to understand the sign "New Content" in a configuration page of a CMS, the user needs to have a previous idea of what a user is.

III. Procedural Semantics - It links the semiotic unit, including its meaning, to the interactive dialogue and the context in which it is being used. It takes into account all the different pragmatic meanings that users should correctly guess in order to interpret a sign in the way intended by the designer.

IV. Referential Content - It represents the real world concepts that a sign refers to. For instance, the sign "New Banner" could refer to the real world concept of a banner and its meaning.

V. Interactive Function - It represents how the user interacts with a web sign and the purpose of the sign. For instance, a text which changes colour when a user hovers the mouse pointer over it; it is a navigational sign that represents a link that the user can click to display additional content.

VI. Dialogic Function - It is the navigational-dialogic function of a sign in the context of the dialogue being performed between a user and web interface. For instance, the dialogic function of a button "Back" could refer to a content that has not been visited in the website by the user or it could refer to content that has been already visited.

VII. Ontologies - It is the set of concepts and skills that the user should own for understanding web semiotic units and what they want to communicate.

Speroni (2006) places the concept of ontology in the process of communication between web user and designer/website and argues the following: from the web user (receiver) perspective, an ontology is the "corpus" of knowledge that needs to be mastered in order to correctly interpret the meaning of web semiotic units. From the designer/website (sender) perspective, it can be asserted that an ontology is the "corpus" of knowledge, which is presupposed and pointed out by semiotic units.

The W-SIDE model defines the following ontologies:

- Interlocutor/Institution Ontology (ILO) - It refers to the knowledge related to the institution/corporation/organisation on behalf of which a website talks. It also includes the knowledge related to the generic sector to which the institution/corporation/organisation belongs.

- Topic Ontology (TO) - It represents the knowledge related to the particular topic/subject the interlocutor talks about.

- Context Ontology (CO) - It represents the knowledge that is not directly related to the website's topic but relevant for making the dialogue possible and comprehensible.

- Website Ontology (WO) - It represents the knowledge or conventions that are generated in the website itself.

- Internet Ontology (IO) - It represents all the knowledge related to concepts, skills and conventions shared among people that are familiar with web browsing in general.

- Web Domain Ontology (WDO) - It represents the knowledge that is shared among websites that belong to the same domain or "business sector".

- Common Sense Ontology (CSO) - It represents the set of concepts that belong to the common sense (common and everyday terms that users need to master in order to correctly interpret semiotic units). 
Table 1 defines the levels of familiarity of users with ontologies. Speroni (2006) states that these values were obtained using empirical validation.

\begin{tabular}{|l|l|}
\hline Level of Familiarity & Value \\
\hline High & 9 \\
\hline Medium & 6 \\
\hline Low & 3 \\
\hline
\end{tabular}

Table 1: Level of familiarity of user profiles with ontologies

This section explains how the Ontology Translation expands the W-SIDE Model defining a process that can be applied to the content management interface of a CMS.

To formalise the Ontology Translation process, assumptions regarding user profiles and how the content is structured in the CMS are needed. Subsequently, these assumptions are used for establishing conditions when the concept is viable and for its validation.

\section{SEMIOTICS AND CMS}

\subsection{ASSUMPTIONS}

User Profiles Using the context of what a CMS is, two types of user profiles are identified: technical users and non-technical users. These two profiles are formulated as follows: technical users are in charge of the technicalities of the CMS, and they are well trained to setup and configure software. Non-technical users are in charge of managing the content that is published in a website, and they have to possess a high level of understanding of the website's topic. In order to formalise the expanded model, these two profiles need to be defined in terms of the W-SIDE Model. This formal definition lets establish the level of knowledge that each profile has regarding the ontologies that are being used in the CMS's content management interface. The formalised user profiles are defined in the following list using the evaluation scale of user familiarity with ontologies described in Table 1.

- System Administrator - It is the user who is in charge of the installation, configuration, security and updated of the CMS. Table 2 describes the System Administrator profile in terms of its familiarity with the ontologies defined in Section 2.2.

\begin{tabular}{|l|l|l|}
\hline Ontology & Level of Familiarity & Value \\
\hline Interlocutor & Medium & 6 \\
\hline Topic & Low & 3 \\
\hline Context & Medium & 6 \\
\hline Website & High & 9 \\
\hline Internet & High & 9 \\
\hline Web Domain & Low & 3 \\
\hline Common Sense & High & 9 \\
\hline
\end{tabular}


- Website Content Manager - It is the user who is in charge of maintaining, creating and updating website content. Table 3 describes the Website Content Manager profile in terms of its familiarity with the ontologies defined in Section 2.2.

\begin{tabular}{|l|l|l|}
\hline Ontology & Level of Familiarity & Value \\
\hline Interlocutor & High & 9 \\
\hline Topic & High & 9 \\
\hline Context & Low & 3 \\
\hline Website & Low & 3 \\
\hline Internet & Low & 3 \\
\hline Web Domain & Low & 3 \\
\hline Common Sense & High & 9 \\
\hline
\end{tabular}

Table 3: Website Content Manager Profile

Contrasting the description of both the System Administrator profile and the Website Content Manager, the following facts can be highlighted: While the System Administrator has a high level of familiarity with the ontologies that are directly related to the "world" of the Internet and CMSs, their level of familiarity with the ontologies related to the topic of the website that is being administrated by the CMS is low. (b) Website Content Manager has a high level of familiarity with the ontologies related to the topic that the website talks about. However, their level of familiarity with the ontologies related to the Internet and CMSs is low. Based on facts (a) and (b) the following can be predicted: (I) Managing the content of the website is not a trivial task neither from the perspective of the System Administrator nor from the perspective of the Website Content Manager. (II) It is unlikely that the Website Content Manager will be able to manage in a straightforward manner the website content without help/training from the System Administrator. (III) Even after training, the Website Content Manager may be subject to confusion and misunderstandings. Especially given the fact that it is highly probable that the language of the semiotic units in the CMS administration interface are incompatible with the topic that the website is talking about.

CMS Content Structure It is assumed that the CMS structures content in the following manner: A website is composed of sections. Sections may contain subsections; sections also may have content. Subsections may contain other subsections and also each subsection may have content. Subsections are recursive entities, which means a subsection of a subsection can have another subsection. Finally, content can be composed of text, images, links, forms, flash files, flash videos, JavaScript carousels, etc.

\subsection{EXPANDED MODEL - ONTOLOGY TRANSLATION CONCEPT}

W-SIDE Model is composed of seven concepts: semiotic unit, conceptual semantics, procedural semantics, referential content, interactive function, dialogic function, and ontology. The proposed expanded model incorporates an extra concept: "Ontology Translation".

In the context of CMS, Dirgahayu, Setiani and Zukhri (2014) argue that organisations must define information requirements before publishing content on their websites. The information requirements constitute the information to be published and its organisation. It must be pointed out that information organisation does not refer to either how web pages are linked to each other or how web pages should look like. It defines in which web page an information item should be presented (in relation with other information items) taking into account the levels of importance of all the information items that need to be published on the website. 


\section{Based on the concept of information organisation and the generic nature of the structure of the CMS content structure, Villamarin (2014, p. 39) defines the Ontology Translation concept as follows: "Ontology Translation is the process of establishing an alternative web semiotic unit for signs that belong to a different ontology that the user needs to interpret."}

The Ontology Translation process requires that both the information organisation and the CMS content structure have been previously established. Ontology translation may be performed by the System Administrator with the help of the Website Content Manager; by combining their user profiles a new profile can be defined: Ontology Translator.

According to Villamarin (2014), the Ontology Translation process is composed of the following steps:

I. Definition of information organisation - It may be carried out by the business side of the organisation that owns the website. It may be performed with the help of the Website Content Manager. It requires a high knowledge of the business domain.

II. Definition of CMS content structure - It may be performed by the System Administrator (or a technician). It is required an advance level of expertise in web technologies.

III. Selection of key signs - It may be performed by an Ontology Translator or by the System Administrator with the help of the Website Content Manager. The System Administrator selects the signs that can be mapped from the CMS content structure to the information organisation. The Website Content Manager selects the signs that could lead to confusion when new content is added to the website. These signs mainly refer - but they are not limited to - the Website and Internet ontologies.

IV. Definition of alternative web semiotic units - It may be carried out by the Website Content Manager with the help of the System Administrator. Alternative web semiotic units are defined for the key signs previously selected (Step III); its main purpose is to help avoid communication breakdowns during application-user interaction. These signs mainly refer to the interlocutor or topic ontologies.

V. Application of alternative web semiotic units - It may be performed by the System Administrator; it consists of wrapping the key signs selected in Step II with the alternative web semiotic units from Step IV. This step may include altering the CMS's source code using appropriate programming techniques, i.e. JavaScript functions, PHP controllers, etc.

It is expected that after performing the Ontology Translation process communication breakdowns during application-user interaction will decrease. Figure 1 depicts user's interpretation of signs after ontology translation has been performed. 


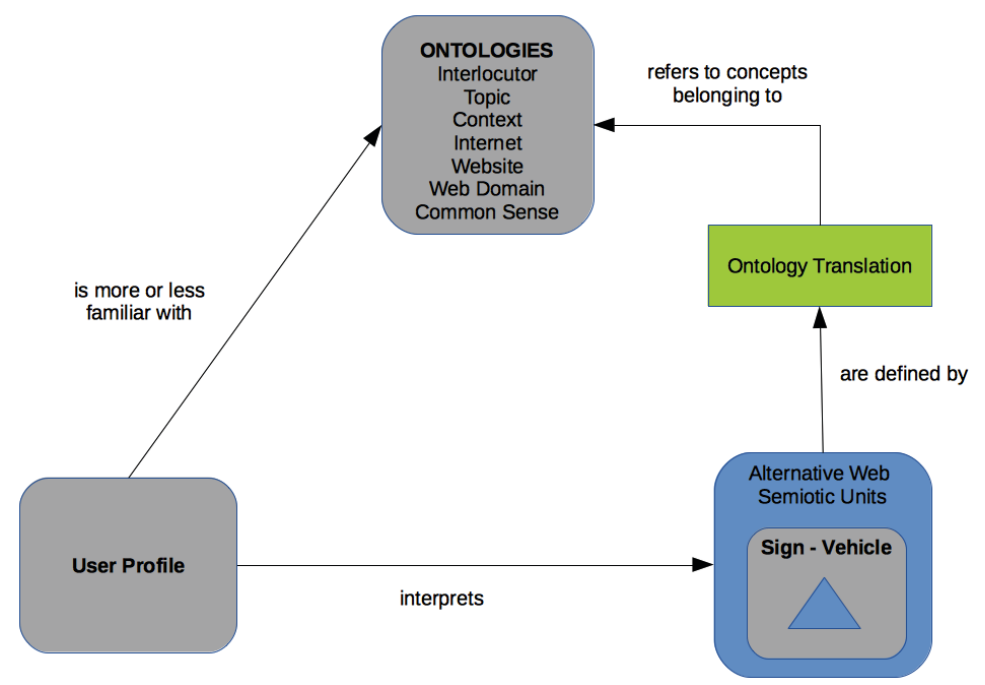

Figure 1: User sign interpretation after ontology translation

\section{PROOF OF CONCEPT}

An experimental approach was used to demonstrate the feasibility of the Ontology Translation concept. The website www.ferech.com, which is the personal website of a fashion designer based in Prague, was taken as a guideline to define a fictitious scenario. Using the fictitious scenario an experiment was designed. The main subject of the experiment was the Website Content Manager.

\subsection{FICTITIOUS SCENARIO}

As the main objective of the scenario is to manage a fictitious website that should serve as a personal catalogue for a fashion designer, both the ILO and the TO refer to the world of "fashion design".

The information organisation is designed as follows: the website is composed of works of the fashion designer; there are three types of works (footwear, fashion design, and accessories). Footwear is composed of collections; each collection is composed of type of customers (i.e. women, men, etc.). Each type of customer contains models and each model has a description (i.e. text, images, videos, etc.). Fashion design is composed of collections; each collection is composed of types of customers (i.e. women, men, etc.). Each type of customer contains garments and each garment has a description (i.e. text, images, videos, etc.). Accessories is composed of types of accessories. For the fictitious scenario just one type is defined: bags. Bags contains collections, each collection is composed of types of customers (i.e. women, men, etc.). Each type of customer contains models and each model has a description (i.e. text, images, videos, etc.).

The CMS content structure consists of a website that is composed of three sections, each section is composed of subsections and each subsection can be composed of more subsections. Sections and subsections may include content. 


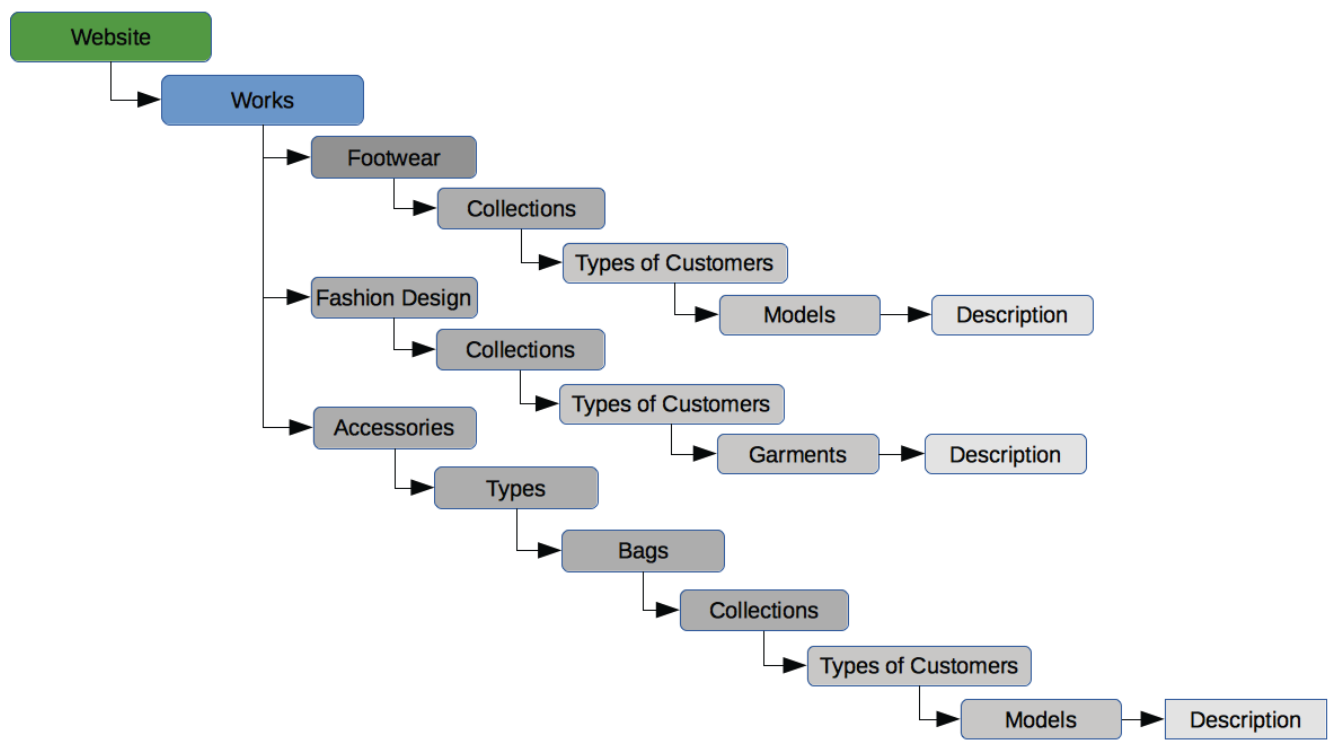

Figure 2: Information organisation fictitious scenario

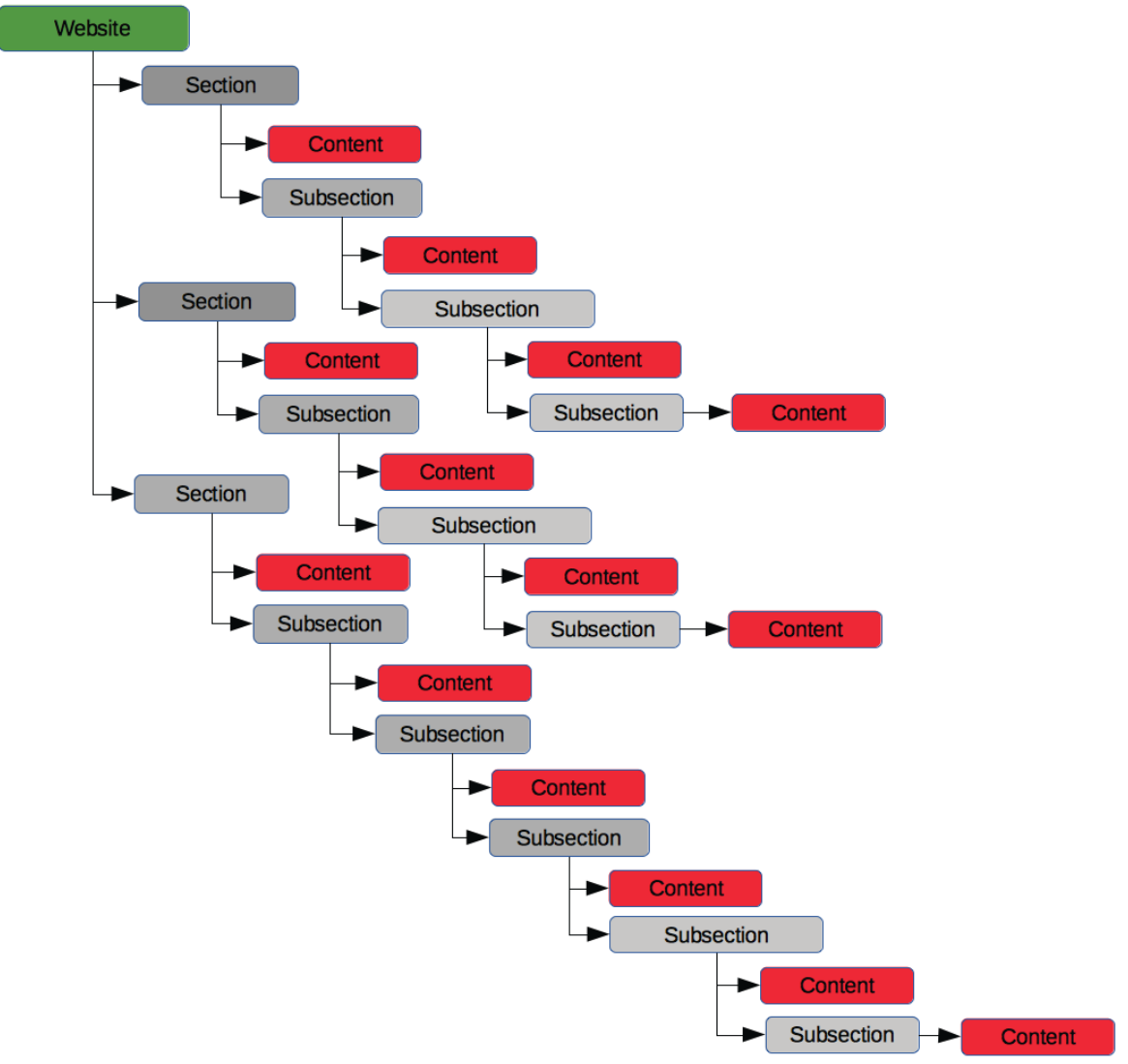

Figure 3: CMS content structure 
Figure 2 and Figure 3 depict the information organisation and the CMS content structure of the fictitious scenario. By comparing both structures it is noticeable that information organisation and CMS content structure are compatible. Consequently, a mapping from Website Ontology and Internet Ontology to Interlocutor Ontology and Topic Ontology is plausible.

\subsection{VALIDATION OF THE ONTOLOGY TRANSLATION CONCEPT}

After defining the information organisation and the CMS content structure, steps I and II of the Ontology Translation process have been completed. The remaining steps require a working CMS in order to be performed.

Validation of the Ontology Translation concept was carried out by building a working prototype. The prototype was developed using the source code of the Open Source CMS WicaWeb available at https:// github.com/mushoq/wicaweb. Mushoq (2015) points out that WicaWeb is built using Zend Framework an open source framework for developing web applications using PHP programming language; being this the main reason why WicaWeb was selected from among other CMSs like Joomla or WordPress. Adding a new functionality to it, it is equivalent to create or modify a module, controller, helper, or function on a Zend Framework web application.

Top-level requirements for the prototype were established from the definition of both the Ontology Translation process and the fictitious scenario. Two top level requirements were identified: (1) Systems Administrator should be able to generate tooltips (alternative web semiotic units) for specific labels, buttons, and links of the CMS's content administration interface. (2) Website Content Managers should be able to visualise the tooltips (alternative web semiotic units) generated by the System Administrator.

The source code of the prototype is available at https://github.com/ AlexandrosV/WicaWeb. According to Mushoq (2015), the minimum software requirements to run the WicaWeb are: PHP version 5.3, Apache HTTP server 2.2, and MySql 5.5. The prototype contains a new module called Ontology, which fulfills toplevel requirements (I) and (II). A detailed technical description of the prototype is given by Villamarin (2015) in section 3.3 of his Master's project The Semiotic Engineering of Content Management Systems. Figure 2 shows the Ontology module in the control panel of the WicaWeb CMS.

\subsection{SELECTION OF KEY SIGNS \& DEFINITION OF ALTERNATIVE WEB SEMIOTIC UNITS}

Four generic configuration interfaces can be found in the WicaWeb's CMS module: (1) configuration interface for sections, (2) configuration interface for subsections, (3) configuration interface for contents, and (4) forms for creation of new sections, articles content and link to content.

Based on Section 4.1, Table 4 defines the selected keys and their alternative web semiotic units for the generic interface (1).

\begin{tabular}{|l|l|}
\hline Sign & Alternative Web Semiotic Unit \\
\hline New Section & New Work \\
\hline Sections & Work \\
\hline Section name & Work Name \\
\hline
\end{tabular}

Table 4: Alternative web semiotic units for generic interface (1) 
Table 5 defines the selected keys and their alternative web semiotic units for the generic interface (2). Table 6 defines the selected keys and their alternative web semiotic units for the generic interface (3). Table 7 defines the selected keys and their alternative web semiotic units for the generic interface (4).

\begin{tabular}{|l|l|}
\hline Sign & Alternative Web Semiotic Unit \\
\hline New Section & New Collection \\
\hline New Content & New Description \\
\hline Subsections & Collection \\
\hline Subsection name & Collection name \\
\hline Has Subsections & Has Type of Customer \\
\hline
\end{tabular}

Table 5: Alternative web semiotic units for generic interface (2)

\begin{tabular}{|l|l|}
\hline Sign & Alternative Web Semiotic Unit \\
\hline New Content & New Description \\
\hline Contents & Description \\
\hline Content & Collection name \\
\hline
\end{tabular}

Table 6: Alternative web semiotic units for generic interface (3)

\begin{tabular}{|l|l|}
\hline Sign & Alternative Web Semiotic Unit \\
\hline New Section & * Same as for generic interface (2) \\
\hline New Content & * Same as for generic interface (2) \\
\hline New subsection of ... & $\begin{array}{l}\text { * New Type of Customer (in the case of Fashion Design sub- } \\
\text { section) }\end{array}$ \\
\hline
\end{tabular}

Table 7: Alternative web semiotic units for generic interface (4)

\subsection{APPLICATION OF ALTERNATIVE WEB SEMIOTIC UNITS}

It constitutes the last step the Ontology Translation process. It is performed by the System Administrator using the administration interface for the Ontology module.

\subsection{TEST CASES}

To evaluate the prototype as well as the Ontology Translation process the following two test cases are defined: (1) Without applying any alternative web semiotic units of the footwear section and subsections, the Website Content Manager must create a new model of shoes (Textura Kids) for a new collection (Textura Spring Summer 2015) for new type of customer (kids). A short description (text) of the new model should be added. (2) Alternative web semiotic units are applied to all the selected signs of the Fashion Design section and subsections. The Website Content Manager must create a new garment (radio) for a new collection (Volumetra Sprint Summer 2015) for a new type of customer (men). A short description (text) of the new garment should be added.

A short introduction (five minutes approximately) about the WicaWeb is given to the Website Content Manager before performing the test cases. After the test cases are carried out, feedback is gathered using a simple questionnaire. The questionnaire serves as an instrument to attempt to quantify the usefulness of applying alternative web semiotic units in the WicaWeb content's management interface. 


\subsection{QUESTIONNAIRE}

It is composed of ten closed-ended questions. Answer are quantified using a matrix that contains a rating scale of five points (adverbs: very, slightly, neither, slightly, and very) and pairs of adjectives to measure attributes. Each question intents to quantify one of the following attributes: level of complexity (adjectives: easy and difficult) level of understanding (adjectives: clear and vague), level of assertion (adjectives: clear and confusing) and level of helpfulness (adjectives: helpful and useless). Table 8 shows the questions and its corresponding main purpose.

\begin{tabular}{|c|c|}
\hline Question & Purpose \\
\hline Estimate the level of difficulty of completing task 1. & $\begin{array}{l}\text { Measure the level of complexity of adding new content to the } \\
\text { website without using the Ontology Translation concept. }\end{array}$ \\
\hline $\begin{array}{l}\text { How understandable is the meaning of the table } \\
\text { headers Section and Subsection when adding the } \\
\text { required content? }\end{array}$ & $\begin{array}{l}\text { Measure the user's understanding level of the default inter- } \\
\text { face for managing content in the WicaWeb. }\end{array}$ \\
\hline $\begin{array}{l}\text { How understandable is the purpose of the buttons New } \\
\text { Section and New Content afters reading their labels? }\end{array}$ & $\begin{array}{l}\text { Measure the user's understanding level of the purpose of the } \\
\text { default buttons for managing content in the WicaWeb. }\end{array}$ \\
\hline $\begin{array}{l}\text { When adding the description of the model of shoes, } \\
\text { was it clear which button must be used to add the } \\
\text { description? }\end{array}$ & $\begin{array}{l}\text { Measure the user's assertion level when deciding which but- } \\
\text { ton is used for a specific action. }\end{array}$ \\
\hline Estimate the level of difficulty of completing task 2. & $\begin{array}{l}\text { Measure the level of complexity of adding new content to the } \\
\text { website after applying the Ontology Translation concept. }\end{array}$ \\
\hline $\begin{array}{l}\text { How understandable is the meaning of the table } \\
\text { headers Collection, Type of Customer, Garment, and } \\
\text { Description when adding the required content? }\end{array}$ & $\begin{array}{l}\text { Measure the user's understanding level of the interface for } \\
\text { managing content in the WicaWeb after applying the Ontol- } \\
\text { ogy Translation concept. }\end{array}$ \\
\hline $\begin{array}{l}\text { How understandable is the purpose of the buttons } \\
\text { New Collection, New Type of Customer, New Gar- } \\
\text { ment, and New Description after reading their labels? }\end{array}$ & $\begin{array}{l}\text { Measure the user's understanding level of the purpose of key } \\
\text { buttons for managing content in the WicaWeb after applying } \\
\text { the Ontology Translation concept. }\end{array}$ \\
\hline $\begin{array}{l}\text { When adding a description of a garment, was it clear } \\
\text { which button must be used to add a description? }\end{array}$ & $\begin{array}{l}\text { Measure the user's assertion level when deciding which but- } \\
\text { ton is used for a specific action after applying the Ontology } \\
\text { Translation concept. }\end{array}$ \\
\hline $\begin{array}{l}\text { Estimate how helpful were the table headers Collec- } \\
\text { tion, Type of Customer, Garment, and Description } \\
\text { when adding the required content. }\end{array}$ & $\begin{array}{l}\text { Measure the level of helpfulness of applying the Ontology } \\
\text { Translation process over table headers in the content man- } \\
\text { agement interface. }\end{array}$ \\
\hline $\begin{array}{l}\text { Estimate how helpful were the button's labels New } \\
\text { Collection, New Type of Customer, New Garment, and } \\
\text { New Description when adding the required content. }\end{array}$ & $\begin{array}{l}\text { Measures the level of helpfulness of applying the Ontology } \\
\text { Translation process over key buttons in the content manage- } \\
\text { ment interface. }\end{array}$ \\
\hline
\end{tabular}

Table 8: Questionnaire

Additionally, the questionnaire includes a section titled Notes, Comments, Remarks that allows the Website Content Manager to express their opinion/experience regarding the two test cases.

\subsection{RESULTS}

In order to complete the proof of concept of the Ontology Translation process, both test cases were carried out by a fashion designer based in Prague. The fashion designer fulfils the profile of a Website Content Manager. After analysing the results of the questionnaire, the following assertions can be made.

For users whose level of familiarity with the Context, Website, Internet, and Web Domain Ontologies is low, managing website content may be a difficult task even if the organisation information and the CMS Content Structure have been previously defined. Table headers like: Section and Subsection in combination with button's labels like: New Section, New Content, etc., may cause confusion during application-user interaction. 
For instance, the button's label New Section and the table header Subsections may produce communication breakdowns when adding content to the website. It is mentioned by the fashion designer that because of the table header indicates that subsections are being created, they expected to have a button called New Subsection to create new content. Figure 4 shows this inconsistency in the user's interface.

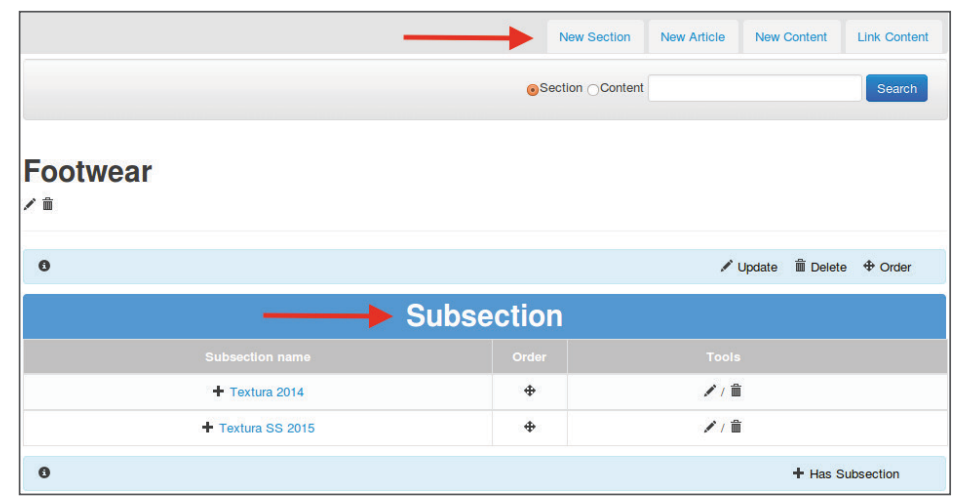

Figure 4: User's interface inconsistency

Also the purpose of buttons with labels such as New Section, New Article, New Content may be not clear and lead to confusion when creating new content for the website. Translating signs from the Context, Website, Internet, and Web Domain Ontologies to Topic and Interlocutor Ontologies facilitates managing website content. By using specific table headers i.e. Collection, Type of Customer, Garment, etc. in combination with buttons labels like New Collection, New Type of Customer, New Garment, etc. helps to reduce communication breakdowns during application-user interaction. Overall, it can be stated that the Ontology Translation concept improves the usability of generic CMSs.

\subsection{CRITICAL EVALUATION}

Assumptions defined in Section 4.1 allowed the Ontology Translation process to be tested and proved. At the same time, they pose a constraint for the concept, and specifically the assumption regarding the CMS content structure. A hierarchical structure composed of sections, subsections, and content is needed in order to define which alternative web semiotic units shall be used at different levels of hierarchy in the information organisation.

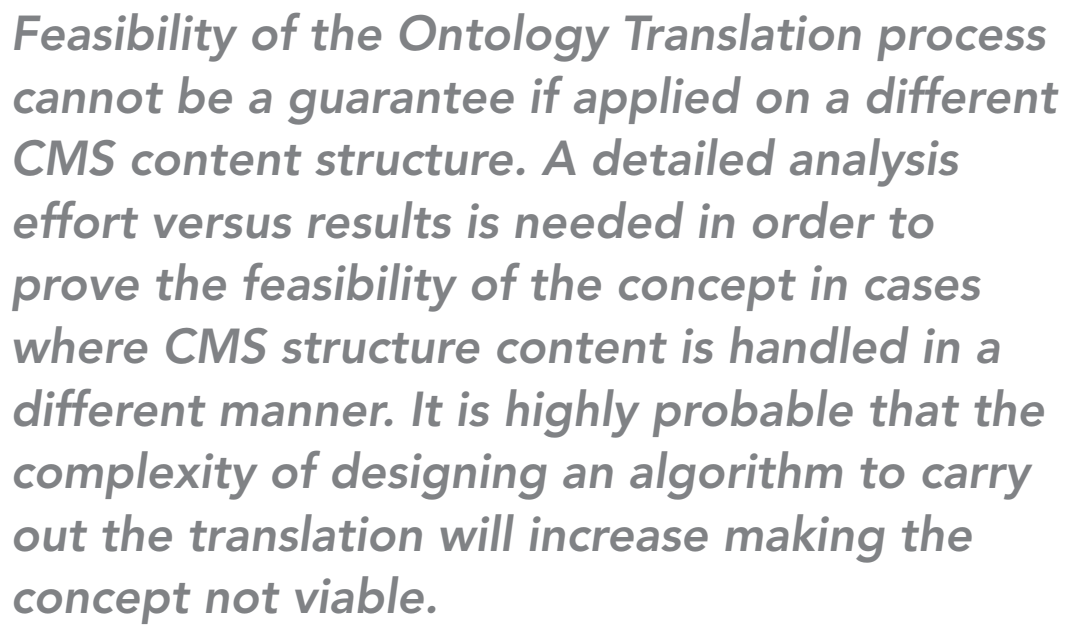

Due to time and logistic constraints, proof of concept of the Ontology Translation concept was limited to just one fictitious scenario. Further validation of the concept is needed in order to strictly prove its feasibility. 


\section{CONCLUSIONS}

Based on the results of the test cases, some of Speroni (2006) can be reasserted. It was demonstrated that the more there is matching between ontologies used in a website and the one mastered by the user, the better the user's understanding and interpretation of signs contained in the interface. It can be also reasserted that to correctly evaluate web semiotic units, user interface language designers should take into account both the user's presupposed knowledge and the knowledge that is directly referred to by the semiotic unit. Not properly considering either of both can lead to misleading assessments of the web semiotic unit. It is also imperative to always keep in mind that regardless of the referential content of a semiotic unit, a sign can have interactive and dialogic functions.

The Ontology Translation concept applied to a CMS prototype contributes in a practical manner to the enhancement of the theoretical semiotic concept W-SIDE model. It also constitutes a practical example of how applying semiotic theory the usability of a CMS's content configuration interface can be greatly enhanced. Furthermore, the validation process of the Ontology Translation concept exposed an extra utility for it; alternative web semiotic units can serve as a guideline for the next action that the user should perform when managing content (this depending on how alternative web semiotic units are presented to the user i.e. marked with a different colour).

Overall, the present project constitutes a step forward to the study of how communication breakdowns may occur when using certain ontology in content management interfaces. It also contributes to the study of how to design better technical interfaces for users that lack the proper knowledge to correctly interpret its signs. Interface designers can use the prototype as a tool to evaluate the efficiency of ontologies used in content management interfaces.

Despite the fact that some important limitations were identified during the critical evaluation of the Ontology Translation process, these limitations should be considered as opportunities for future studies. The main limitations of this project were the size of the survey sampling and the evaluation of the social and cultural implications related to the users' origin. Also, it must be pointed out that the present project did not take into account any aesthetic aspect of the interfaces.

\section{REFERENCES}

Armour, P.G. (2000) 'The Business of Software: The Case for a New Business Model', Commun.ACM, 43(8), pp. 19-22.

De Souza, C.S. (1993) 'The semiotic engineering of user interface languages', International Journal of Man-Machine Studies, 39(5), pp. 753-773

De Souza, C.S., Barbosa, S.D.J. and Prates, R.O. (2001) 'A semiotic engineering approach to user interface design', Knowledge-Based Systems, 14(8), pp. 461-465.

Dirgahayu, T., Setiani, N. and Zukhri, Z. (2014) 'Information requirements engineering for specific content management systems', Open Systems (ICOS), 2014 IEEE Conference on. Oct., pp. 54-59.

Mushoq. (2015) Descargas Available at: http://www.wicaweb.com (Accessed: 11 March 2015).

Speroni, M. (2006) Mastering the Semiotics of Information-Intensive Web Interfaces. University of Lugano.

Valtolina, S., Barricelli, B.R. and Dittrich, Y. (2012) 'Participatory knowledge- management design: A semiotic approach', Journal of Visual Languages er Computing, 23(2), pp. 103.

Villamarin, M.F.A. (2015) The Semiotic Engineering of Content Management Systems. MSc. Teesside University. 